\title{
Refining Relationships
}

\author{
Douglas Sellinger
}

A s a parent, I am enjoying refining my relationship with my daughter as she develops from an adolescent to an amazing young woman. It is her goal to enter a school of pharmacy shortly, and I look forward to becoming colleagues in addition to father and daughter. As in any family, our relationship is constantly changing, sometimes easily and sometimes with struggles.

The relationship between the Canadian Society of Hospital Pharmacists (CSHP) and the Canadian Pharmacists Association (CPhA), two of Canada's premier pharmacist advocacy organizations, is also constantly evolving, characterized occasionally by struggles, yet strongly collaborative at other times.

The new CSHP bylaws, passed at the Society's Annual General Meeting in August 2013, came into effect on May 1, 2014. The Canada Not-for-profit Corporations Act and associated regulations have changed how not-for-profit corporations are structured and behave. These regulatory changes also affect many of our strategic external partners such as $\mathrm{CPhA}$. As part of its own compliance review, $\mathrm{CPhA}$ is taking the opportunity to renew itself. The Association is redefining its membership, governance, and board structure, as introduced to members at the CPhA 2013 Annual General Meeting. These changes will affect $\mathrm{CPhA}$ 's relationships with its external partners, including CSHP.

Last August, the CSHP Executive invited the CPhA Chief Executive Officer and President to our national Council Issues Day to present the proposed CPhA membership model. With this new model, $\mathrm{CPhA}$ will shift from individually held memberships to organizational membership. Provincial and national pharmacy associations will form the membership of $\mathrm{CPhA}$, with individual members of those associations receiving $\mathrm{CPhA}$ member benefits through their respective organizational memberships. CSHP has been asked to become a member organization of $\mathrm{CPhA}$. Council has not yet committed itself to this course of action; it is still seeking further clarification on the benefits and costs of $\mathrm{CPhA}$ membership to determine if this is something CSHP members will view as beneficial overall.

As national advocates for pharmacists and pharmacy, CSHP and CPhA have partnered on many initiatives, including the ADAPT program, the Blueprint for Pharmacy, the Primary Care PSN (Pharmacy Specialty Network), and more recently a number of drug shortages initiatives. CSHP and $\mathrm{CPhA}$ have worked together with other strategic partners on the creation of the Canadian Drug Shortage Database (www.drugshortages.ca) and on the Multi-Stakeholder Steering Committee on Drug Shortages in Canada (which approved the Protocol for the Notification and Communication of Drug Shortages and A Toolkit for Improved Understanding and Transparency of Drug Shortage Response in Canada). As well, CSHP has partnered with CPhA on member surveys to understand and make public the impact that drug shortages are having on patients and pharmacists.

Regardless of the changes at $\mathrm{CPhA}$ and whether or not CSHP decides to become a member organization of CPhA, we will continue to consider the Canadian Pharmacists Association among our key strategic partners and we look forward to continued collaboration with $\mathrm{CPhA}$ when pharmacist concerns align for our two great organizations.

Douglas Sellinger, BSP, MALT, is Past President and External Liaison for the Canadian Society of Hospital Pharmacists. 